\title{
Announcing the Canadian Journal of Health Technologies - A New Journal From CADTH
}

\section{Key Messages}

- The COVID-19 pandemic has put a spotlight on science and reaffirmed the value of evidence in health care decision-making.

- The Canadian Journal of Health Technologies will publish CADTH work in a single, PubMedindexed, online location, making it easier for our health system partners to search and find CADTH work.

- CADTH is a major Canadian publisher of evidence, advice, and recommendations regarding the assessment and management of health technologies.

- Through the Canadian Journal of Health Technologies, CADTH will expand its reach and its collaborations with producers and users of health technology assessments.

The COVID-19 pandemic has put a spotlight on science and reaffirmed the value of evidence in health care decision-making. In this environment, CADTH is incredibly proud to launch the Canadian Journal of Health Technologies.

The Canadian Journal of Health Technologies is an open access journal that allows CADTH to publish its work in a standardized manner and in a single, PubMed-indexed, online location. The journal's clearly defined platform addresses known difficulties in systematically searching for and accessing our work.

CADTH is a major Canadian scientific publisher. We're a trusted source of evidence, advice, and recommendations regarding the management of drugs, diagnostics, and medical, surgical, and dental devices and procedures. The Canadian Journal of Health Technologies will feature CADTH's horizon scans, reimbursement reviews, and health technology review reports, and it will be used to publish the proceedings and abstracts from the CADTH Symposium.

The CADTH Symposium is an annual conference for producers and users of health technology assessments. Rich discussions take place at the Symposium but these discussions are not widely shared beyond the Symposium's participants. Publication in the journal will help expand these conversations and provide Symposium presenters with a permanent, searchable citation that will enhance the accessibility of their work.

We're also looking forward to using the journal's Viewpoint and Editorial sections to increase collaboration with our health system partners and stakeholders - particularly decision-makers and patient groups - and more broadly communicate a range of perspectives on health technology management.

The launching of a new Canadian scientific journal is an important milestone in the evolution of CADTH. We welcome your feedback and suggestions.

Nicole Mittmann 